\title{
Le dépôt de l'âge du Bronze moyen de Keravel en Lannilis (Finistère)
}

The Keravel Middle Bronze Age Hoard, Lannilis (Finistère)

Jean-François Villard

\section{OpenEdition}

\section{Journals}

Édition électronique

URL : https://journals.openedition.org/rao/800

DOI : $10.4000 /$ rao. 800

ISBN : 978-2-7535-1609-0

ISSN : $1775-3732$

\section{Éditeur}

Presses universitaires de Rennes

\section{Édition imprimée}

Date de publication : 31 décembre 2009

Pagination : 43-55

ISBN : 978-2-7535-1086-9

ISSN : 0767-709X

\section{Référence électronique}

Jean-François Villard, « Le dépôt de l'âge du Bronze moyen de Keravel en Lannilis (Finistère) », Revue archéologique de l'Ouest [En ligne], 26 | 2009, mis en ligne le 31 décembre 2011, consulté le 22 août 2022. URL : http://journals.openedition.org/rao/800 ; DOI : https://doi.org/10.4000/rao.800 


\title{
Le dépôt de l'âge du Bronze moyen de Keravel en Lannilis (Finistère)
}

\author{
The Keravel Middle Bronze Age Hoard, Lannilis (Finistère)
}

\author{
Jean-François VILLARD*
}

\begin{abstract}
Résumé : Le dépôt du Bronze moyen de Keravel en Lannilis (Finistère) fut mis au jour lors d'une opération de diagnostic archéologique en 2007. Une dizaine d'objets ou fragments d'objets fut découverte, probable reflet d'un dépôt partiellement détruit par une petite carrière d'arène granitique, creusée à une époque indéterminée. Une pointe de lance décorée et des fragments de haches à talon ou à rebord se rattachent au groupe typologique de Tréboul. Ils sont accompagnés de fragments d'une grande hache de type breton. L'ensemble, datable du Xv siècle avant J.-C., s'insère dans un état des connaissances, déjà richement documenté en Bretagne, à propos de ce type de dépôts.

Abstract: The Middle Bronze Age Keravel hoard, Lannilis (Finistère), was discovered during an archaeological evaluation in 2007. The small assemblage of objects or fragments uncovered is probably reminiscent of a larger hoard partially destroyed by a small disused granite gravel quarry of uncertain date. A decorated spear head and some palstave or flanged-axe fragments are attributed to the Treboul Group. Fragments of a large Breton Type palstave were also present. The assemblage dates from 15 th century $B C$ and adds new material to this already well documented hoard type in Brittany.
\end{abstract}

Mots clés : dépôt, âge du Bronze moyen, hache à talon, hache à rebord, pointe de lance, groupe de Tréboul.

Key words: hoard, Middle Bronze Age, palstave, flanged-axe, spear head, Treboul Group.

\section{INTRODUCTION}

La réalisation d'un diagnostic archéologique en 2007 , sur une douzaine de parcelles à lotir sises sur le territoire de la commune de Lannilis, non loin de la chapelle SaintSébastien, a permis de mettre au jour les restes d'un petit dépôt de l'âge du Bronze composé d'une dizaine de pièces.

\section{Contexte topographique et conditions d'intervention}

Les terrains concernés se situent au sud du bourg, sur un versant dominant des zones humides et un ruisseau affluent de l'Aber Benoît (fig. 1). L'intervention a traité une superficie de cinq hectares.
Géographiquement, la commune de Lannilis se situe à proximité de la côte nord-ouest du Léon (Finistère). Le site est localisé au cœur d'un vaste éperon de $10 \mathrm{~km}$ de long pour $4 \mathrm{~km}$ de large, limité au nord par la ria de l'Aber Wrac'h et au sud par celle de l'Aber Benoît.

Les terrains diagnostiqués constituent une partie du versant exposé au sud, au-delà de la rupture du plateau actuellement occupé par le bourg. L'altitude du secteur étudié varie de 38 à 25 m NGF, avec un pendage général d'environ $4 \%$. Dans la partie basse, le pendage s'accentue jusqu'à près de $10 \%$, s'accompagnant d'une évolution du substrat arénitique vers des bancs de sable gris ou de graviers à mesure que l'on s'approche des zones humides (ruisseau et marécages). Le traitement en profondeur des parcelles par les engins agricoles modernes a accentué l'érosion de la partie supé-

* Responsable d'opération INRAP; Centre de recherche archéologique de Finistère - 2 rue du Stivel, 29000 Quimper. 


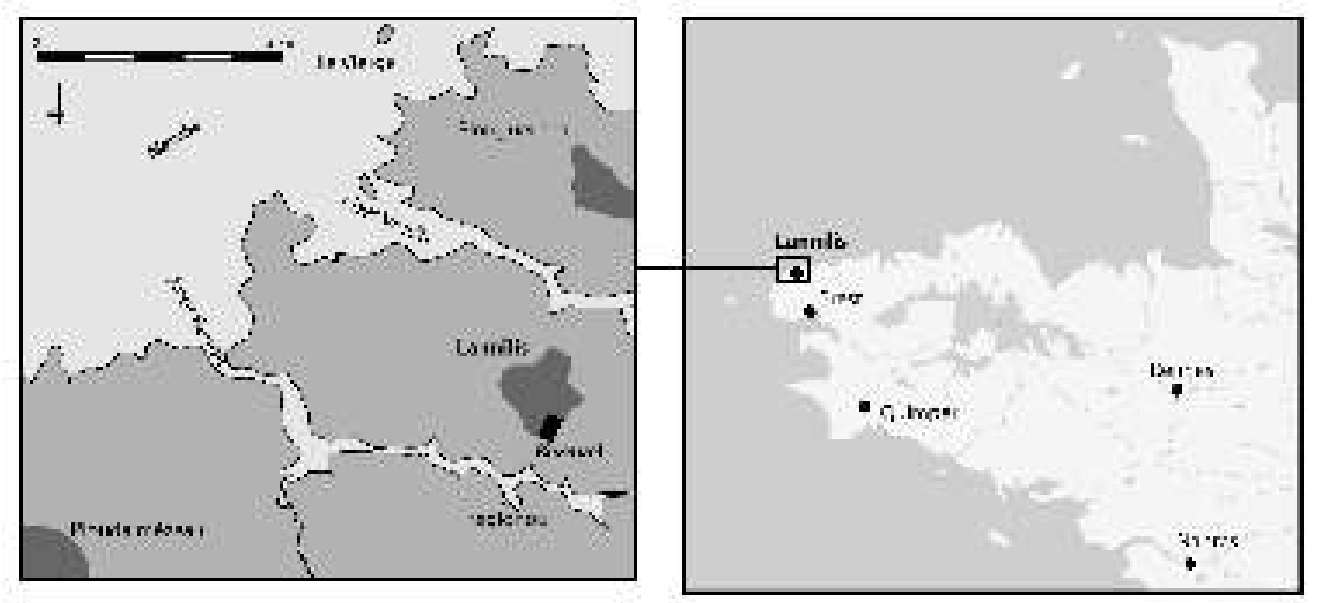

Figure 1: Localisation de la commune de Lannilis et du site de Keravel.

Figure 1: Location map of the of Lannilis district and of the site of Keravel. rieure du substrat et limité ici tout espoir de découvrir du mobilier archéologique erratique ou d'éventuelles structures en élévation.

\section{Le contexte archéologique}

La commune de Lannilis, tout comme la région des Abers en général, recèle un environnement archéologique assez riche et diversifié (Galliou, 1989, p. 190-191; documentation du SRA de Bretagne). Pour les périodes pré- et protohistoriques, douze sites y sont recensés, parmi lesquels une occupation du Néolithique (Roual) et six sépultures de l'âge du Bronze (Kerdrel, Kerscao, Rascol, Kerfrichoux, Prat-arSimon-Pella). Cette dernière, découverte fortuitement quelques mois avant le diagnostic de Keravel (Le Goffic, 2006), a livré un mobilier du Bronze ancien (poignard et hache à légers rebords en bronze, lots de minuscules clous décoratifs en or et pointes de flèches en silex). Une hache à talon, de provenance inconnue et aujourd'hui disparue, fut également recensée sur la commune par J. Briard (Gabillot, 2003, p. 174). L'âge du Fer est représenté par le petit cimetière de Pembrat-Vihan (stèle et urnes). Deux autres stèles sont également connues, plus à l'ouest, à Coum et à Kerguisquin. Enfin, un souterrain (Le Prat) complète le panorama pour l'âge du Fer. Par ailleurs, rappelons que l'occupation ayant livré le célèbre collier en or de Kerellen en Tréglonou se situe de l'autre côté de la ria, sur la rive gauche de l'Aber Benoît, à moins de $5 \mathrm{~km}$ à vol d'oiseau.

Pour l'Antiquité, trois occupations (moulin de Poulfougou, Penhoat et Kergoadou) livrent de la poterie, des tuiles et des fossés. Plusieurs monnaies gallo-romaines, à la provenance indéterminée, sont également répertoriées à Lannilis. En outre, les Abers sont fréquemment cités comme sites portuaires au cours de l'Histoire, notamment durant la période gallo-romaine. Des itinéraires antiques reliant la mer et les sites de Saint-Frégant (villa) et Kérilien-Plouneventer (agglomération secondaire) se suivent au nord du bourg actuel de Lannilis.

Enfin, plusieurs sites médiévaux et d'époque moderne (manoir, motte, enclos...) font le lien avec l'histoire récente. Des textes attestent de l'existence d'un bourg dès 1330 , cœur de la paroisse de Ploudiner, réunissant alors Lannilis, Tréglonou et Broennou avant un éclatement survenu au $\mathrm{xv}^{\mathrm{e}}$ siècle.

\section{Les résultats du diagnostic}

En dépit de ce riche environnement, les résultats du diagnostic furent peu spectaculaires malgré un taux de décapage de plus de $10 \%$ des parcelles (fig. 2). En dehors du dépôt faisant l'objet de cet article, le site révéla un vaste réseau de fossés de parcellaires, parfaitement structuré, à l'orientation décalée de $45^{\circ}$ par rapport au paysage actuel (Villard, 2007). Ce réseau ancien s'accompagne d'un fond de chemin creux. D'autres espaces de circulation ont pu compléter l'ossature de ces parcelles. Le recoupement de certains fossés par des limites parcellaires figurant sur le cadastre de 1841, dont l'orientation est similaire à celle du document actuel, date ce réseau d'une période antérieure au XIX ${ }^{e}$ siècle, sans plus de précision. L'absence totale de mobilier dans les sections fouillées ne permet pas de l'intégrer à une quelconque période historique. L'action en profondeur des techniques agricoles récentes (cf. supra) a par ailleurs éliminé toute possibilité de découvrir des tessons erratiques anciens. Cette érosion anthropique récente a complété une érosion naturelle du versant, sans doute importante mais dont il est difficile d'estimer la puissance et les conséquences sur les vestiges. Seuls deux petits tessons d'allure antique (pâte grise et dégraissant roulé) ont été mis au jour au nord de la tranchée B. XI, hors structures. Ces derniers ont peu de poids parmi 
Figure 2 : Lannilis, Keravel : plan diachronique des vestiges.

Figure 2: Diachronic plan of features.

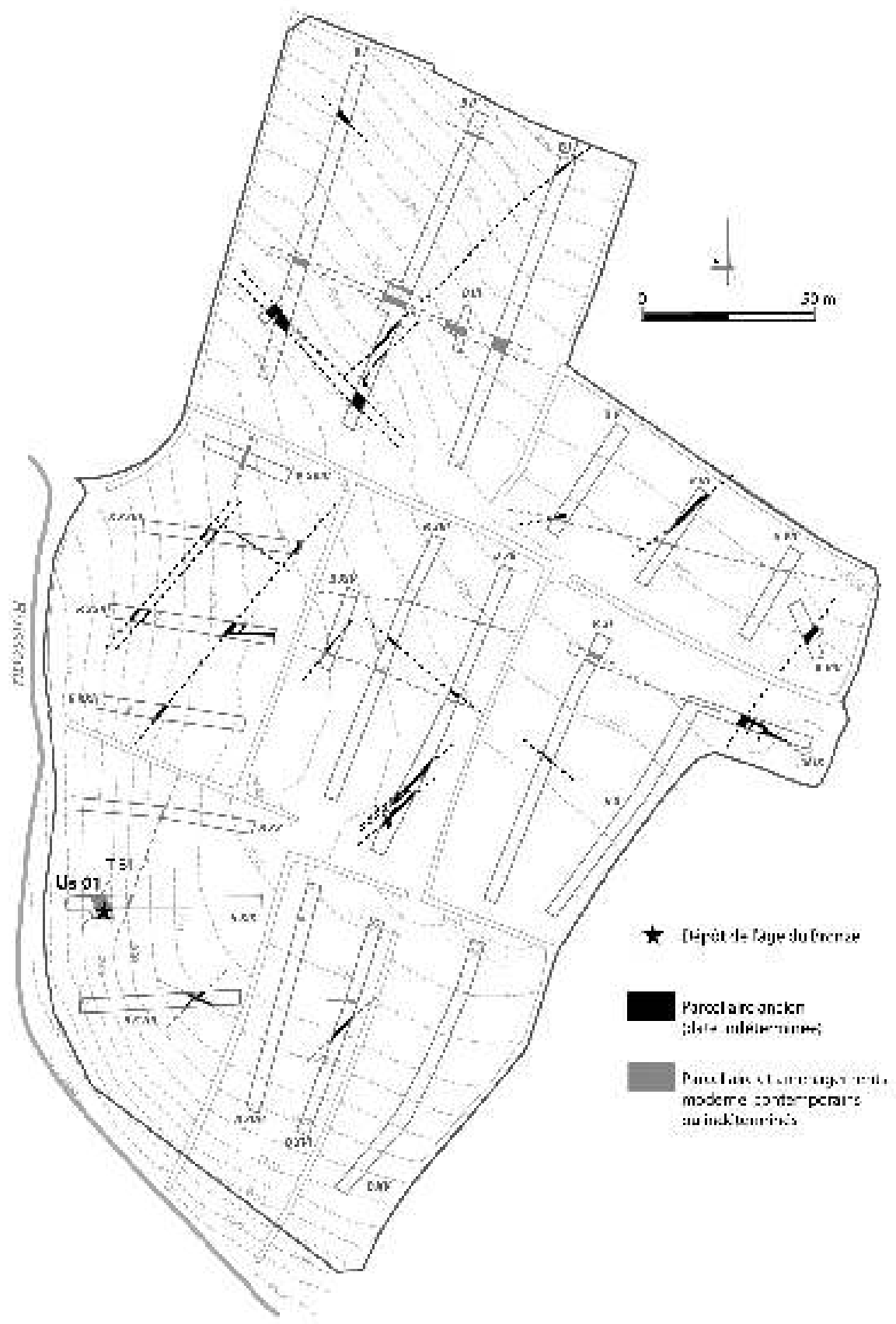

les nombreux fragments de verre, de faïence voire de plastique découverts au même niveau, dans la couche remaniée par l'agriculture, entre la base de la terre végétale et la surface actuelle du substrat sain.

Le profil des fossés, très arasés et conservés en moyenne sur $0,30 \mathrm{~m}$ de profondeur, ainsi que leur appréhension sous forme de segments intermittents, ne favorisent guère l'interprétation du réseau qui se répartit cependant sur les cinq hectares étudiés. On note simplement que la régularité de l'implantation en fait un ensemble cohérent. Ces fossés constituent les vestiges d'un large terroir homogène.
L'archéologie préventive met fréquemment au jour de tels ensembles parcellaires. Ils sont connus dès l'Antiquité autour d'établissements ruraux tels que ceux du Moustoir (Le Bihan et Villard, 2001b; 2003a et b) ou de Kerjaouen (Villard, 2002; Roy, 2008), l'un et l'autre à Quimper. Cependant, la mise en place du bocage à partir des $\mathrm{XIV}^{\mathrm{e}}-\mathrm{XV}^{\mathrm{e}}$ siècles ne simplifie pas l'attribution chronologique de ces réseaux fossoyés. Ce phénomène de bocage, conforme à ce que l'on connait des modes de structuration du paysage en Europe occidentale (Guilaine, 1991), se poursuit ou se modifie, à un rythme parfois rapide, jusqu'aux XVIII ${ }^{e}$-XIX ${ }^{e}$ siècles. Faute de relations entre ce réseau parcellaire et les structures d'habitat 
de ses exploitants, malheureusement absentes, le débat sur sa datation demeure ouvert.

Cette quarantaine de fossés s'accompagne de quelques structures isolées parmi lesquelles les restes d'un petit dépôt d'objets en bronze.

\section{LE DÉPÔT}

\section{Le contexte de découverte}

Le dépôt fut découvert au sud-ouest des parcelles étudiées (tranchée B. XIX), en bas de pente, à proximité du ruisseau affluent de l'Aber Benoît (fig. 3). Ce secteur regroupe deux éléments imbriqués difficilement discernables en surface: d'une part une fosse (Us. 01), d'autre part un lot de dix objets ou fragments d'objets en bronze.

\section{La fosse}

La fosse (Us. 01) présente un plan ovale dont l'axe longitudinal est orienté nord-ouest/sud-est (fig. 4, 5, 6). Elle mesure près de $6 \mathrm{~m}$ de long pour 3,20 $\mathrm{m}$ de large. Elle se situe en dessous de la rupture topographique des $28 \mathrm{~m} \mathrm{NGF}$,

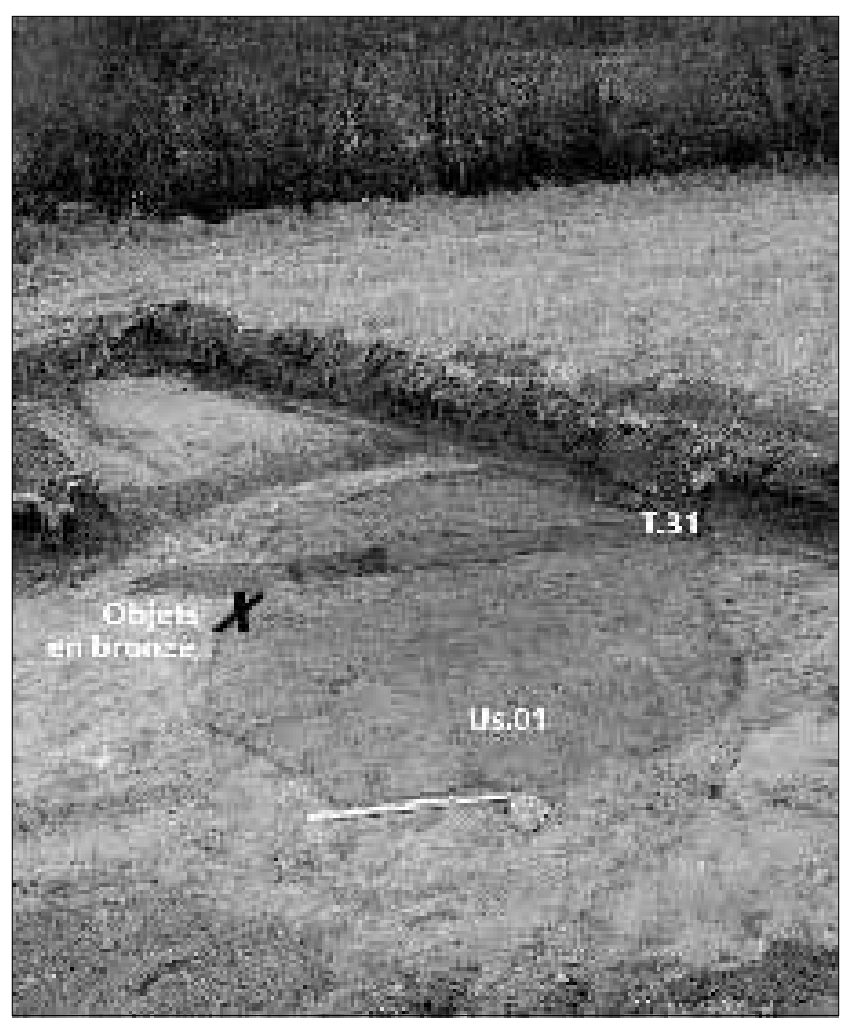

Figure 3 : Vue vers l'ouest de la fosse Us.01 recoupée par le fossé moderne T.31.

Figure 3: The pit US 01 cut by the modern ditch T.31, view toward the west dans un secteur de fort pendage (10\%). Son comblement, de terre arénitique très claire, la distingue peu du substrat environnant. Ce dernier est relativement varié dans cette portion basse du site où les bancs d'arène jaune alternent avec des bancs grisâtres ou des graviers. La fouille partielle de la structure montre un creusement en front de taille vers l'amont. Au nord-ouest, le fond de la fosse présente un fond plat qui se transforme, à contre-pente, en vaste cuvette.

Partiellement incrustés dans le substrat, localement relativement meuble, au niveau du flanc sud-ouest du creusement principal, les objets en bronze étaient groupés sur une superficie de moins d'un demi-mètre carré, en limite extérieure de la fosse. Seules quelques traces extrêmement ténues de poussière verdâtre, apparues au décapage, signalent leur présence.

\section{Interprétation}

La situation particulière de ces objets, en position décentrée et incrustés dans le flanc de la fosse, pose la question de la liaison entre objets et structure. Un temps interprétés comme appartenant à un même ensemble, tant les limites de la fosse Us.01 étaient difficilement repérables en surface, la fouille montra que les deux unités devaient être dissociées. Les bronzes ne sont pas déposés dans la fosse, ils constituent un ensemble distinct et antérieur (fig. 4).

La fosse, avec sa technique de creusement en front de taille, évoque davantage une structure d'extraction de matériaux. Des cas similaires se rencontrent régulièrement lors d'opérations archéologiques; citons pour exemple les carrières d'époques moderne et contemporaine de Mez-Notariou à Ouessant (Le Bihan, 2008), celles médiévales, de la rue Bourg-les-Bourgs à Quimper (Villard, 2005) ou celles protohistoriques, de Penvillers (Le Bihan, 1997), toujours à Quimper. Le contexte géologique de Keravel, avec ses bancs de belle arène, se prêterait sans mal à cette interprétation. La couleur extrêmement claire du comblement conférerait à la structure les critères d'une certaine ancienneté, peut-être même dès l'âge du Bronze, mais sans pour autant la dater avec précision. Rappelons que certains comblements de fossés du parcellaire ancien, découverts sur le site, présentent les mêmes caractéristiques.

$\mathrm{Ni}$ la forme asymétrique du fond de la fosse, ni l'absence totale de mobilier archéologique en dehors des objets en bronze excentrés, n'évoquent une structure funéraire, type de vestiges que l'on était en droit de rencontrer ici compte tenu de l'environnement archéologique de la commune (cf. supra) et qui fut à la base de la prescription du diagnostic. L'homogénéité du remplissage, sans aucune trace d'argile rubéfiée ou de terre charbonneuse, ne cadre pas plus avec une fonction artisanale ou liée à un habitat au demeurant absent du reste des parcelles étudiées. Enfin, ni la position 
Figure 4 : Lannilis, Keravel : Us.01 (B XIX), plan et coupes stratigraphiques. Figure 4: US 01 (BXIX), plan and sections.

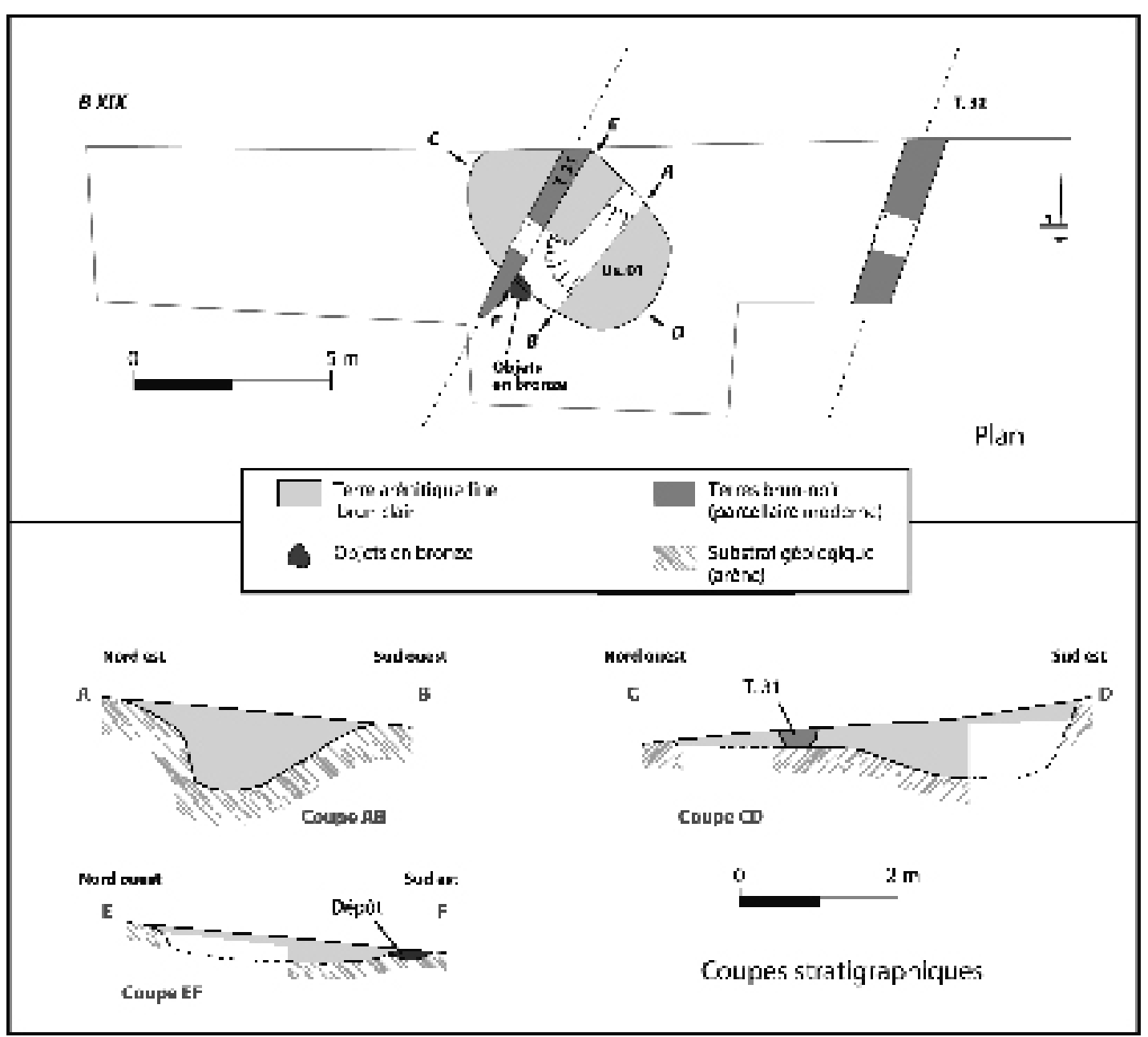

de la fosse, sur un versant à forte pente, et sa taille ne favoriseraient ces dernières fonctions. Cette situation renforcerait plutôt la première hypothèse d'une carrière destinée à l'extraire de l'arène.

Les bronzes ont été, quant à eux, retrouvés dans un secteur restreint, en limite extérieure de l'excavation. Il s'agit ici d'un lot et non d'objets dispersés. L'hypothèse de pièces ayant migré en bas de pente, suite à la destruction par les labours d'une structure archéologique, située en amont, ne s'accorderait pas avec cette concentration. De plus, aucune structure particulière ayant pu receler ces dernières n'a été mise au jour durant le diagnostic. L'hypothèse d'un lot déposé sur ce bas de pente doit être retenue, d'autant que son analyse révèle que les objets se rattachent à des typologies contemporaines (cf. infra). Cependant, aucune trace du creusement initial du dépôt ou de sédiment spécifique n'est discernable autour de ces pièces.

Tout indique que le creusement de la fosse Us.01, à une époque sans doute très ancienne mais indéterminée, lié à la recherche de matériau arénitique, a recoupé, ou tout au moins frôlé, un dépôt de l'âge du Bronze ou ce qu'il en res- tait. L'hypothèse de travaux récents, agricoles ou autres, ayant fait resurgir des objets fut émise mais semble peu en accord avec l'aspect ancien et homogène du sédiment de la fosse. De même, l'idée d'une récupération volontaire ou d'un pillage fut suggérée, les objets subsistants n'étant alors que le reflet d'un dépôt plus important; cependant, la masse de métal laissé sur place $(500 \mathrm{~g})$ ne cadre pas avec un tel cas.

Le creusement du fossé T. 31 à une période récente (seconde moitié $\mathrm{du} \mathrm{xx}^{\mathrm{e}}$ siècle), n'a sans doute pas simplifié le problème. Lui aussi a pu faire resurgir quelques objets anciens et contribuer à la dégradation du dépôt d'origine. Rappelons qu'une hache à talon, de type et de provenance indéterminée et aujourd'hui disparue, fut recensée sur la commune (cf. supra).

\section{Le mobilier du dépôt}

Le dépôt se compose de deux objets en mauvais état ou usés, une pointe de lance et une hache, accompagnés de huit autres fragments (tableau 1). 


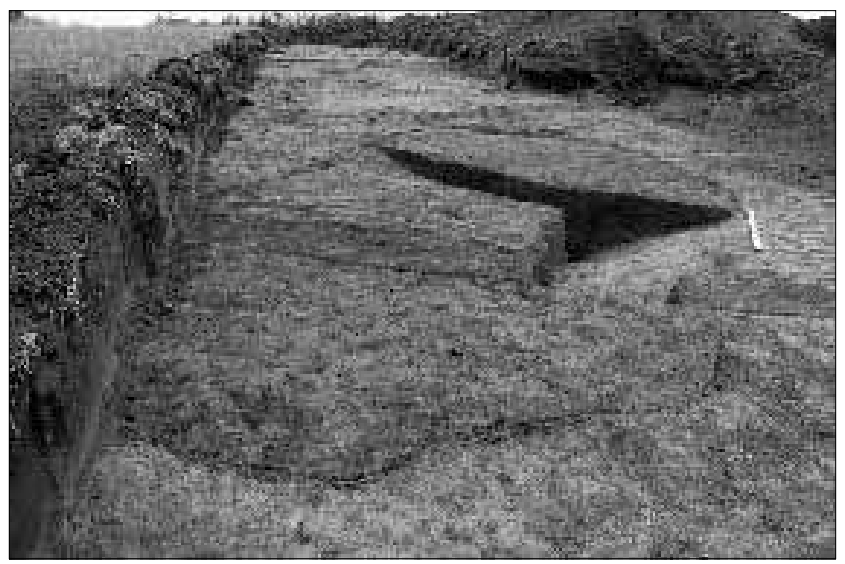

Figure 5 : Vue vers l'est de la fosse Us.01 en fin de fouille. Figure 5: The excavated pit US. 01, view toward the east.

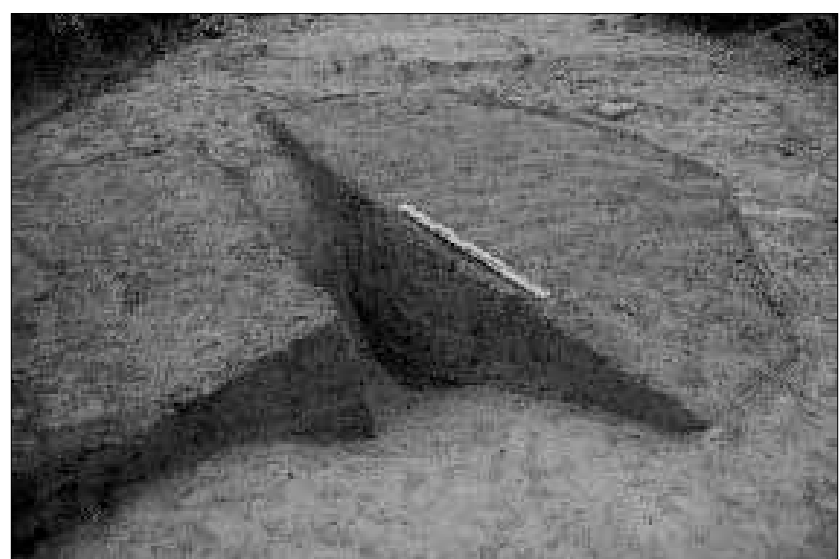

Figure 6 : Vue de détail de la fosse Us 01 et de son comblement arénitique.

Figure 6: Detail of pit US. 01 and its decomposed granite gravel fill.

\begin{tabular}{|c|c|c|c|c|c|c|c|}
\hline OBIT & $\boldsymbol{N}^{*}$ & $\begin{array}{l}\text { Longueur } \\
\text { 'eloin! }\end{array}$ & $\begin{array}{l}\text { Lergeur } \\
\text { len } c r . \text { l }\end{array}$ & $\begin{array}{l}\text { Epaitseur } \\
\text { (ercmi) }\end{array}$ & $\begin{array}{l}\text { Polds } \\
\text { ier. gt? }\end{array}$ & $\begin{array}{c}\text { Couleur desurfacer } \\
\text { de Yalloge }\end{array}$ & $\begin{array}{l}\text { Couleur finter ne et } \\
\text { terture de Tollage }\end{array}$ \\
\hline $\begin{array}{l}\text { Furive } \\
\text { delerise }\end{array}$ & t & 2,0 & 3 & 2,0 trias) & $\operatorname{ses} a$ & $\begin{array}{l}\text { pet lwoll ve } \\
\text { I sse at l. isant }\end{array}$ & ¿a r. vale \\
\hline Fonte & $\bar{z}$ & $\equiv \mathrm{B}$ & $2 ;$ & 0,0 & 21,3 & $\begin{array}{c}\text { vavertsom sere } \\
\text { se: }\end{array}$ & $\begin{array}{c}\text { vertpale } \\
\text { it;orane-t velliats }\end{array}$ \\
\hline $\begin{array}{l}\text { Fet teliscis } \\
\text { خtelen }\end{array}$ & z & $s, w$ & 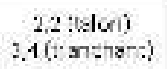 & 2 & $10 \% .2$ & $\begin{array}{l}\text { va.poiles.tlisu } \\
\text { legiserie-tarsr. euse }\end{array}$ & $\therefore$ r. vale \\
\hline $\begin{array}{l}\text { Talon } 5: \\
\text { heche }\end{array}$ & 4 & 3.9 & $2 . ?$ & 1,5 & 206 & issesti_isent & $\begin{array}{c}\text { vertpole } \\
\text { |ejexpme-tedil|ste }\end{array}$ \\
\hline $\begin{array}{l}\text { fismahast de } \\
\text { horte }\end{array}$ & $\Xi$ & $z$ & 3,6 & . & 30,1 & 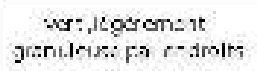 & 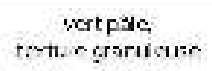 \\
\hline $\begin{array}{l}\text { F/sg d: giance } \\
\text { rachet tzion }\end{array}$ & f.a & $s s$ & 2,9 & 3.9 & 1407 & $\begin{array}{l}\text { brit worbirs oir, } \\
\text { | ssezt_isant }\end{array}$ & $\begin{array}{l}\text { winown, } \\
\text { text_re bulless }\end{array}$ \\
\hline $\begin{array}{l}\text { rizgds giance } \\
\text { rorte:tiolon }\end{array}$ & 62 & 22 & 1,5 & 2 & $+?$ & $\begin{array}{l}\text { vert sotbied -oir, } \\
1=\text { if it I Isnt }\end{array}$ & $\begin{array}{l}\text { vet mogen, } \\
\text { post nhillar: }\end{array}$ \\
\hline $\begin{array}{l}\text { Tisnihant de } \\
\text { lsohes } \\
\text { rebords }\end{array}$ & 7 & ... & 2,9 & 1.16 & 25,9 & wortase & ver $2: 10$ \\
\hline Tige & 8 & 28 & 96 & 0.35 & 2 & Dert & $\begin{array}{c}\text { bertpale } \\
\text { textive pouviese }\end{array}$ \\
\hline Frg inv:estrt, & ب & 2.9 & $z$ & 0.5 & 24.9 & 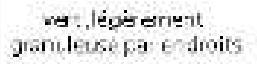 & $\begin{array}{c}\text { vertpide } \\
\text { teoli. egrivise }\end{array}$ \\
\hline
\end{tabular}

Tableau 1 :

Caractéristiques des objets en bronze du dépôt de Keravel. Table 1:

Characteristics of the Middle Bronze Age metalwork of the Keravel's hoard.

\section{La pointe de lance}

La pointe de lance (fig. $7 \mathrm{n}^{\circ} 1$; fig. 8 et 9), est à douille se terminant avant la pointe; sa longueur conservée est de $17,6 \mathrm{~cm}$. Elle est décorée de deux lignes de petits points sur la flamme, au niveau du raccordement des ailerons et de la douille. Ce raccordement est renforcé par deux nervures aplaties longeant cette même douille. Les trous de rivets, aux rebords irréguliers sont de forme oblongue $(1,2 \mathrm{~cm}$ sur 0,7 à $0,8 \mathrm{~cm})$. La douille tronconique est, à sa base, de section elliptique (2,7 x 1,9 cm de diamètre d'emmanchement) avec des arêtes assez nettes dans l'axe de la flamme. Les bords des ailerons sont très abîmes. De même, la base de la douille était brisée et les fragments mêlés aux autres objets formant le lot.

Cette pointe de lance est caractéristique du type de Tréboul, en référence au dépôt découvert en 1948 dans 

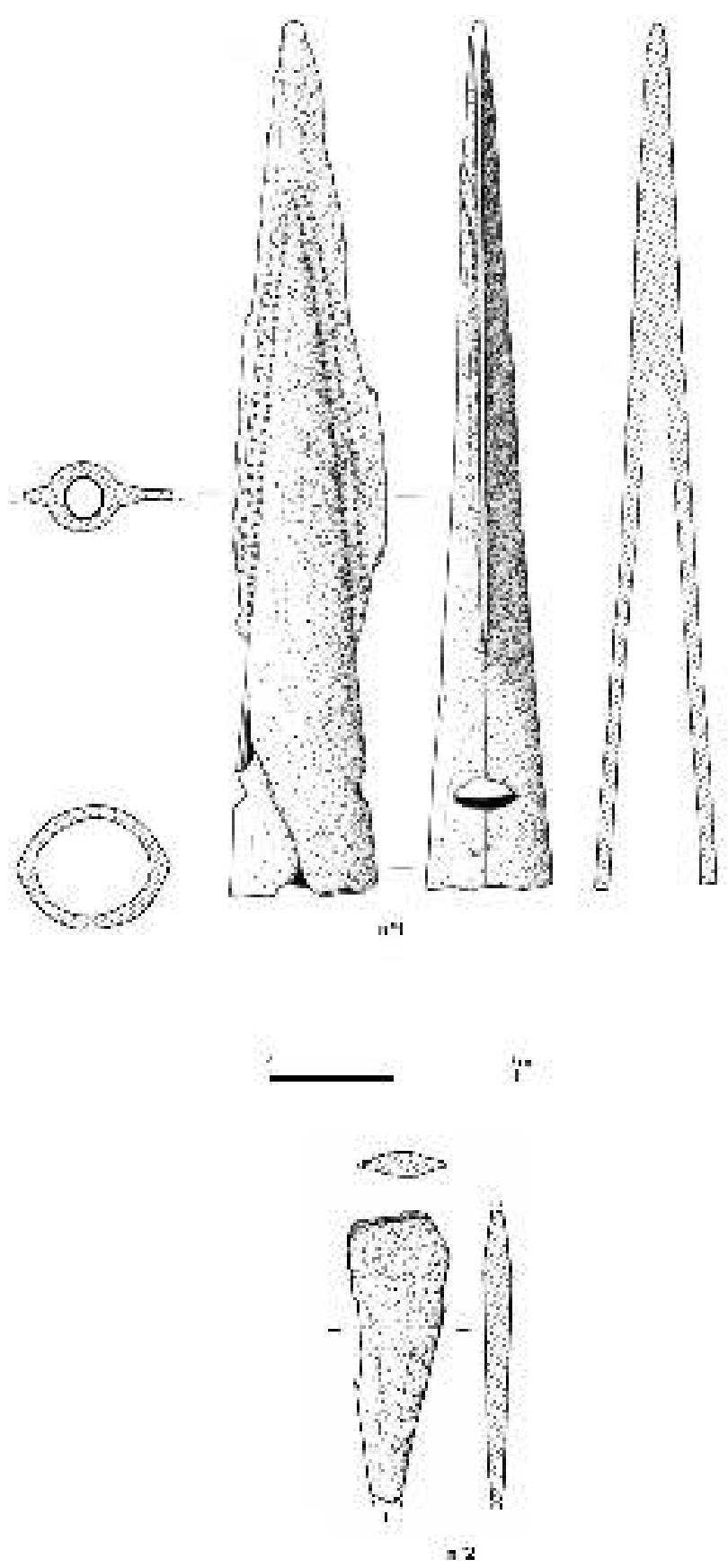

Figure 7 : Lannilis, Keravel : mobilier en bronze, pointe de lance et pointe de lame.

Figure 7: Bronze metalwork, spear head and blade tip.

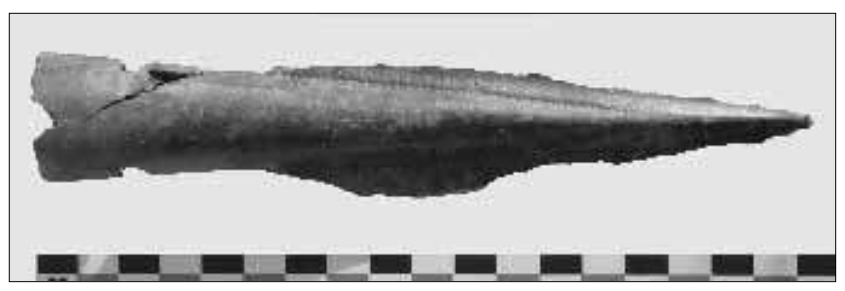

Figure 8 : La pointe de lance du type de Tréboul. Figure 8: Tréboul Type spear head.

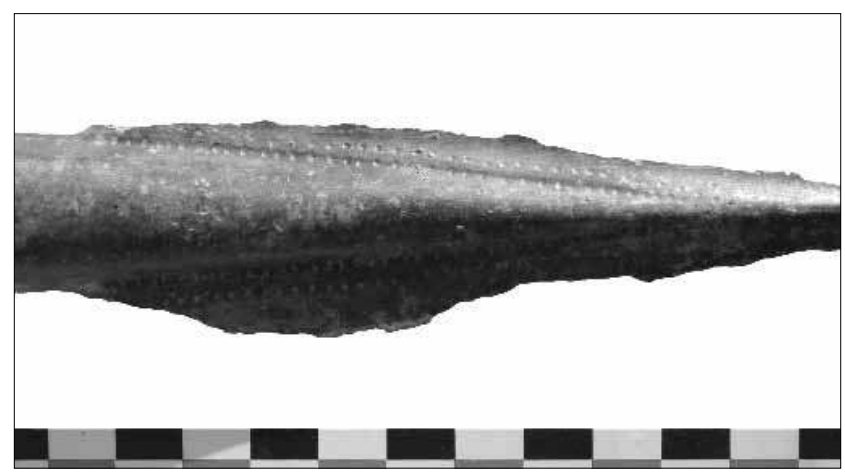

Figure 9: La pointe de lance : détail du décor.

Figure 9: Detail of the decoration on the Tréboul Type spear head.

cette partie de la commune de Douarnenez et qui contenait plusieurs pointes de lances identiques (Briard, 1965; Briard et Mohen, 1983), datées du Bronze moyen. L'exemplaire de Keravel est assez proche du $n^{\circ} 4$ de ce dépôt, avec son décor limité aux ailerons. Elle s'apparenterait au type 52 de la typologie de M. Gabillot (2003). La forme des trous de rivets, aux contours irréguliers, semble indiquer une perforation à froid après moulage, technique que J. Briard date également du Bronze moyen par opposition à celle de la goupille insérée dans le moule lors de la coulée, donnant un trou régulier, et davantage caractéristique du Bronze récent.

\section{Le fragment de pointe de lame}

Le fragment de la pointe d'un objet indéterminé (fig. 7 $\mathrm{n}^{\circ} 2$; fig. 10) dont l'épaisseur s'amenuise vers la base a également été mis au jour. Long de $5,8 \mathrm{~cm}$ pour $2,1 \mathrm{~cm}$ de large, il pourrait s'agir de l'extrémité distale d'une lame de poignard (ou d'épée). Faute d'éléments complémentaires, il est difficile d'être plus précis quant à son attribution typologique.

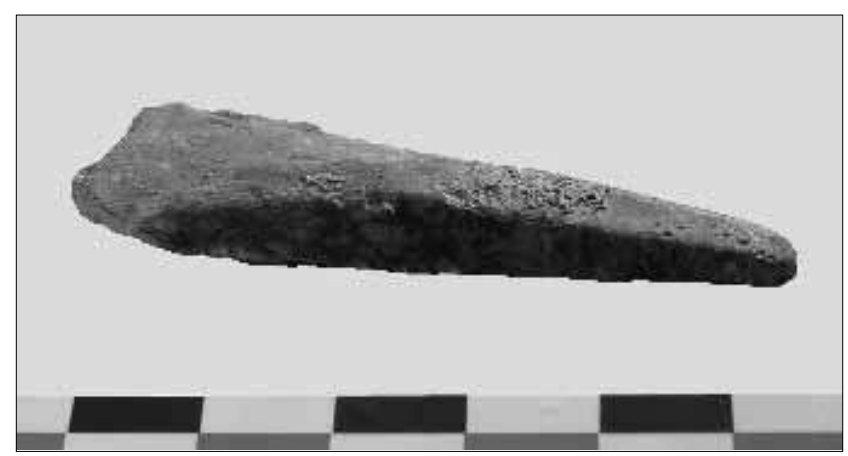

Figure 10 : Pointe de lame $\left(\mathrm{n}^{\circ} 2\right)$.

Figure 10: Blade tip ( $\left.n^{\circ} 2\right)$. 


\section{La petite hache}

La petite hache est du type à talon (fig. 11 ; fig. $12 \mathrm{n}^{\circ} 3$ ). Sa longueur conservée est de $8,85 \mathrm{~cm}$ dont $65 \%$ pour le seul talon. Elle présente une partie proximale à bords très légèrement convexes. La butée, franchement rentrante en section, est légèrement arrondie vue de face. Le profil des larges rebords est biconvexe. Les barbes latérales de coulée sont apparentes mais soigneusement écrasées. La longueur observée de la lame est très courte. Le fil du tranchant est décentré d'environ $4^{\circ}$ par rapport au plan du talon. Le type des rebords encadrant les gorges du talon, avec leur largeur maximale déportée à mi-distance de l'emmanchement et non près de la butée, rattacherait également cette hache aux objets du type de Tréboul (Briard et Verron, 1976), bien que quelques variations de détail existent avec les haches du dépôt éponyme; en particulier, on ne note pas ici de rupture entre le profil convexe des bords et la lame, mais plutôt une continuité. Généralement ce type de hache possède une lame relativement longue et large (cf. infra, fig. 16). La petite taille de celle de l'exemplaire de Keravel pourrait être due à une usure exagérée suite à de nombreux réaffûtages, ce qui expliquerait le désaxage du tranchant; il s'agirait alors d'une hache "en bout de course", devenue non fonctionnelle. Cependant, on n'observe pas de traces d'usure nettes sur la peau du bronze au niveau de la lame. Par ailleurs, compte

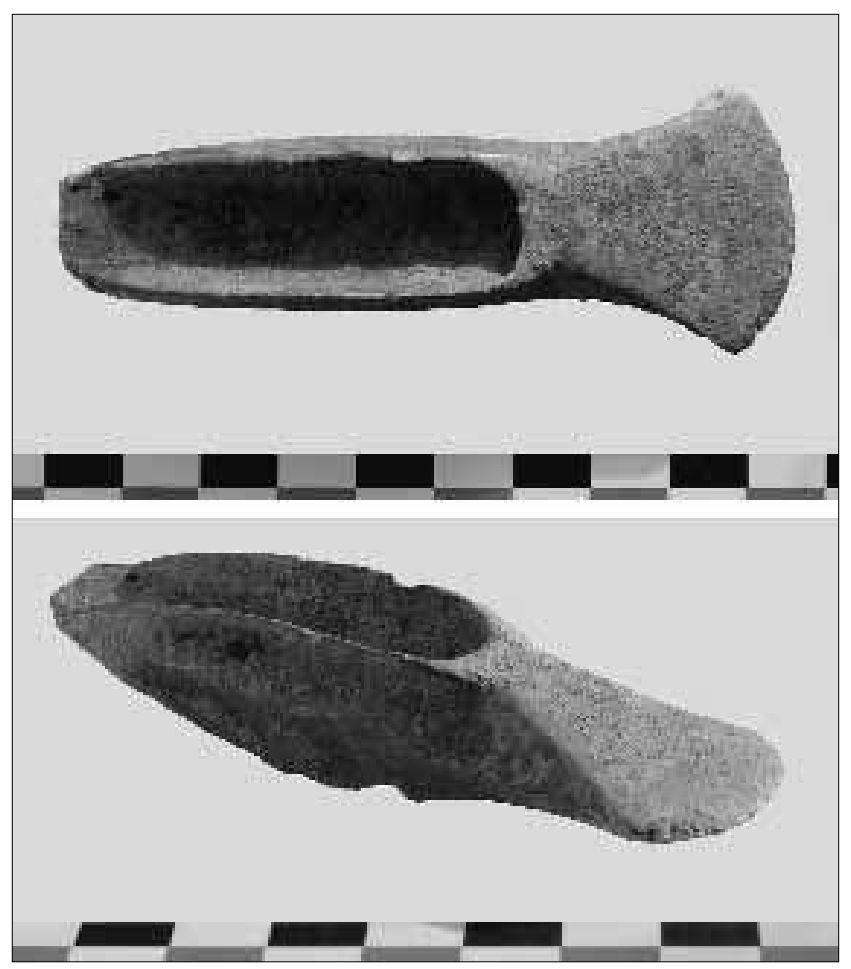

Figure 11 : Hache à talon $\left(\mathrm{n}^{\circ} 3\right)$.

Figure 11: Palstave (n'3).
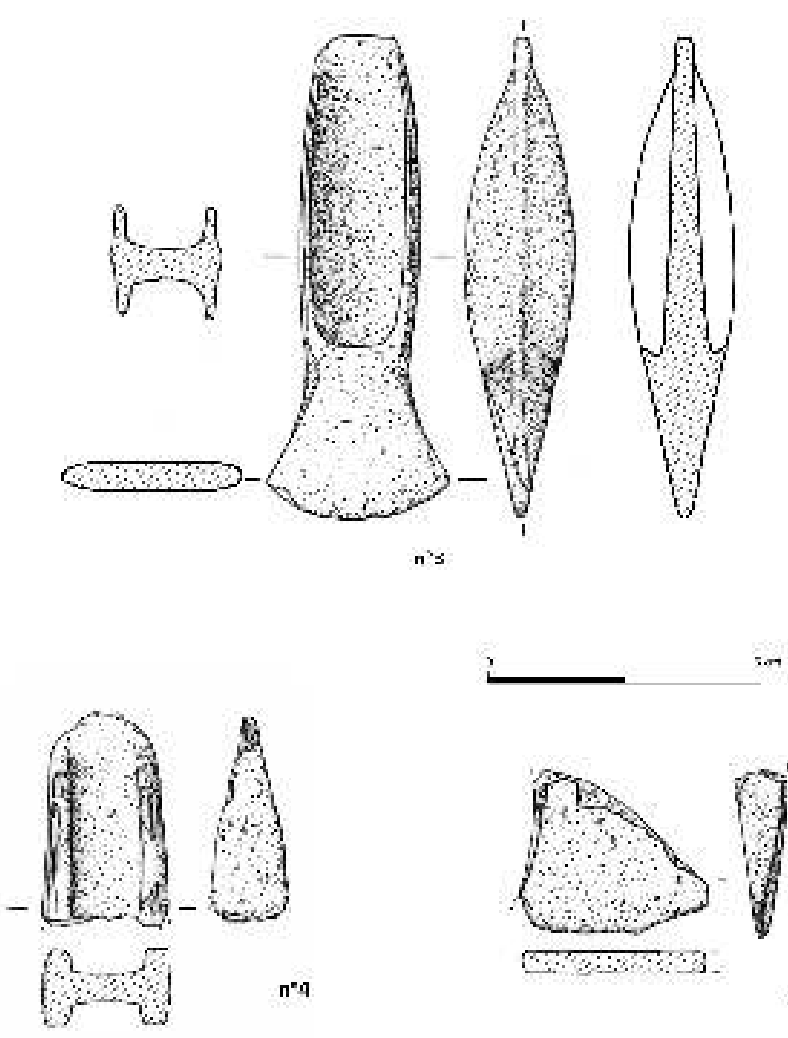

Figure 12 : Lannilis, Keravel : mobilier en bronze, hache à talon et fragments de haches à talon.

Figure 12: Bronze metalwork, ,palstave and palstave fragments.

tenu de la forte concavité des bords de cette dernière, leur prolongement hypothétique supposerait un tranchant extrêmement large, plus de quatre fois supérieur à la largeur du talon si l'on se base sur des exemplaires complets. Le cas d'une large hache n'est pourtant pas exclu d'autant que les individus de grande taille sont fréquents dans le groupe de Tréboul. De plus, dans l'hypothèse de réaffûtages successifs, ceux-ci ont pu être réalisés par martelage, opération induisant inévitablement un élargissement du tranchant. L'examen à la binoculaire de la surface de ce dernier ne révèle cependant la présence que de rares stries, selon différents axes, dont on ne peut affirmer l'origine (aiguisage, utilisation, séjour en milieu arénitique...).

L'hypothèse d'un outil coulé à l'origine avec une lame courte, peut-être pour un usage particulier, doit également être évoquée même si l'on ne connaît pas d'objets de ce type.

L'un des intérêts de cette hache concerne sa technique de réalisation. En effet, l'angle aigu (environ $60^{\circ}$ ) formé par le rentrant de la butée par rapport à l'âme du talon implique une maîtrise particulière de conception des moules. Avec un moule bivalve, ces parties rentrantes gênent le démoulage 
en formant une " contre-dépouille ». Il faut donc soit un moule fractionné, soit une jonction des valves permettant un démoulage oblique selon un angle inférieur à celui du rentrant, soit un moule perdu (en argile par exemple). Ce type de rentrant a pour avantage de renforcer l'emmanchement, les éléments de ce dernier étant maintenus par effet de coin contre l'âme du talon lors des chocs liés à son utilisation.

Ces rentrants ne semblent pas être très courants. En revanche, ils sont la preuve d'une certaine évolution technique des bronziers l'ayant réalisé. Malheureusement, en l'absence de coupes longitudinales sur de nombreux dessins publiés, il n'est pas possible de tirer des conclusions statistiquement pertinentes sur l'apparition et la diffusion de ce caractère. Ce type de rentrant s'observe à l'occasion sur quelques haches à talon normandes (à dépression triangulaire, à décor de sabot fendu et nervure, divers) des dépôts de Verneuil-l'Étang et Heuqueville (Seine-maritime), Saint-George-du-Vièvre et La-Chapelle-du-Bois-des-Faulx (Eure) ou sur des haches du Bassin parisien à Mareil-Marly (Yvelines) et Sucy-en-Brie (Val-de-Marne), mais il ne s'agit là que d'un reflet de l'iconographie disponible (Gabillot, 2003). Ces dépôts sont cependant tous datés de la seconde moitié du Bronze moyen.

\section{Les fragments d'une grande hache}

Les objets $\mathrm{n}^{\circ} 6 \mathrm{a}$ et $\mathrm{b}$ sont probablement deux fragments d'une même grande hache (fig. $13 \mathrm{n}^{\circ}$ 6; fig. 14). Ils ont la même patine de surface, ainsi que la même texture interne bulleuse du bronze. Il s'agit de la partie mésiale et d'un fragment de bord de tranchant d'une forte hache à talon. La butée du talon est arrondie et soulignée par un petit bourrelet. L'amorce d'une nervure centrale, sur le plat de la lame, se raccorde à ce bourrelet. Les flancs sont ornés de stries en chevrons ou en épis. Ce type d'ornementation figure, par exemple, sur une hache mise au jour à dans le dépôt finistérien de Pouldergat (Briard et Onnée, 1971; Giot et al., 1995). Par rapport à la petite hache précédente ( $\left.\mathrm{n}^{\circ} 3\right)$, ce modèle se rattache davantage au groupe des haches à talon de type breton à nervure centrale (Briard et Verron, 1976). Il est cependant intéressant de noter qu'un point commun relie ces deux pièces, à savoir l'angle aigu de la butée. Les remarques technologiques faites pour la hache $\mathrm{n}^{\circ} 3$ sont également valables ici.

\section{Les autres fragments}

\section{Ce sont :}

- un fragment de talon de hache qui, compte tenu de sa taille, est difficilement rattachable à une typologie précise : hache à rebord ou à talon (fig. $12 \mathrm{n}^{\circ}$ 4, fig. 15);

- un fragment de tranchant de hache à talon d'un type indéterminable (fig. $12 \mathrm{n}^{\circ} 5$; fig. 15);

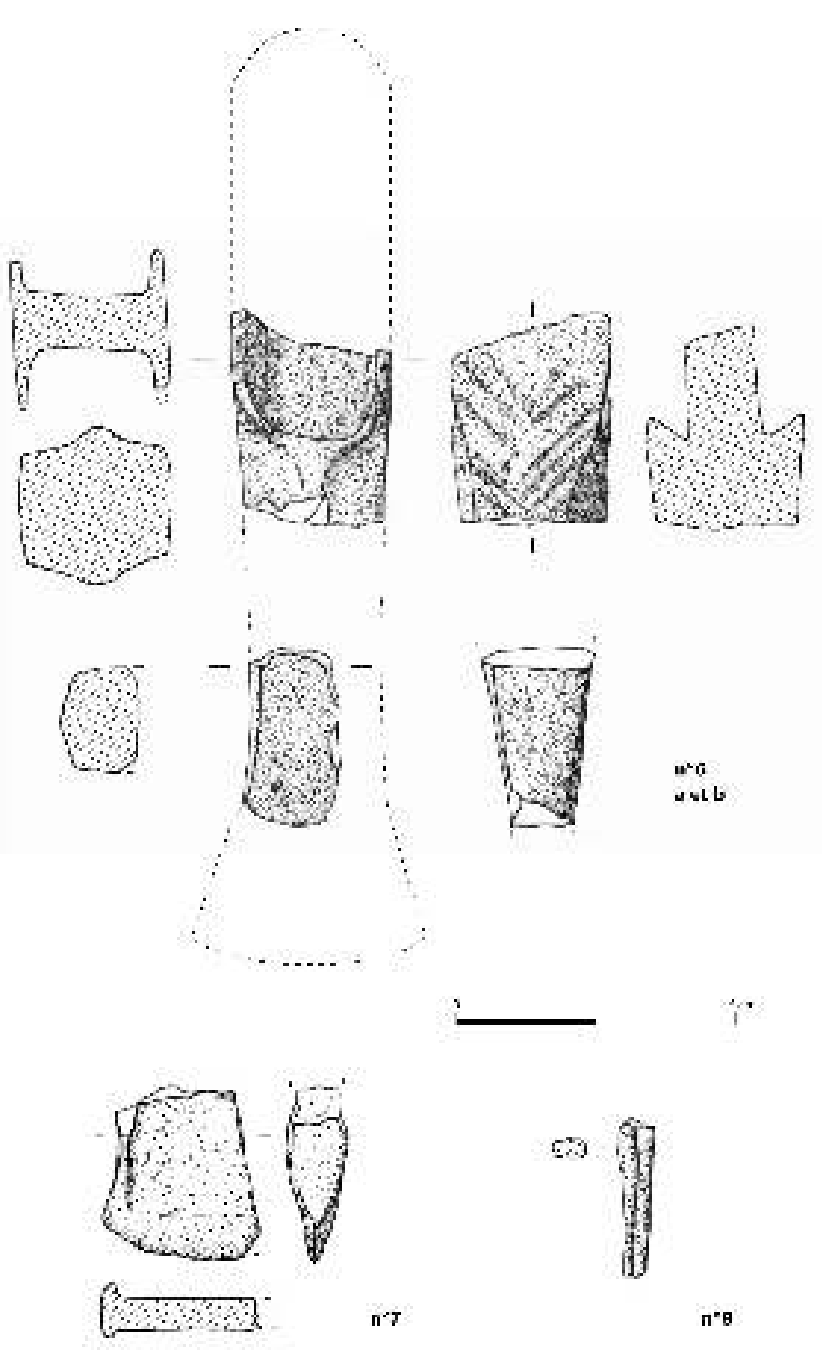

Figure 13 : Lannilis, Keravel : mobilier en bronze, fragments de haches à talon ou à rebord et tige.

Figure 13: Bronze metalwork, palstave or flanged-axe fragments and rod.

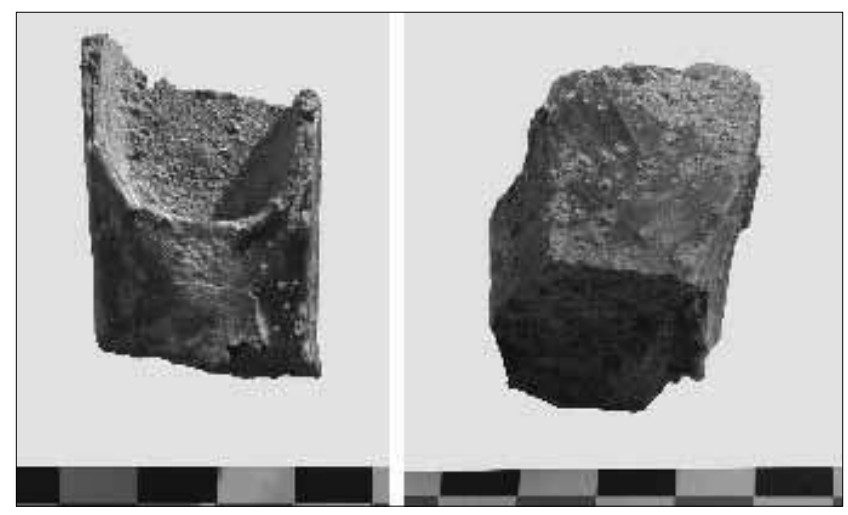

Figure 14 : Fragment de hache à talon n ${ }^{\circ} 6$ a (à gauche) et détail du décor en chevrons sur les flancs (à droite).

Figure 14: Palstave fragment $n^{\circ} \mathrm{C}$ (Left) and detail of the sides herringbone pattern (Right). 


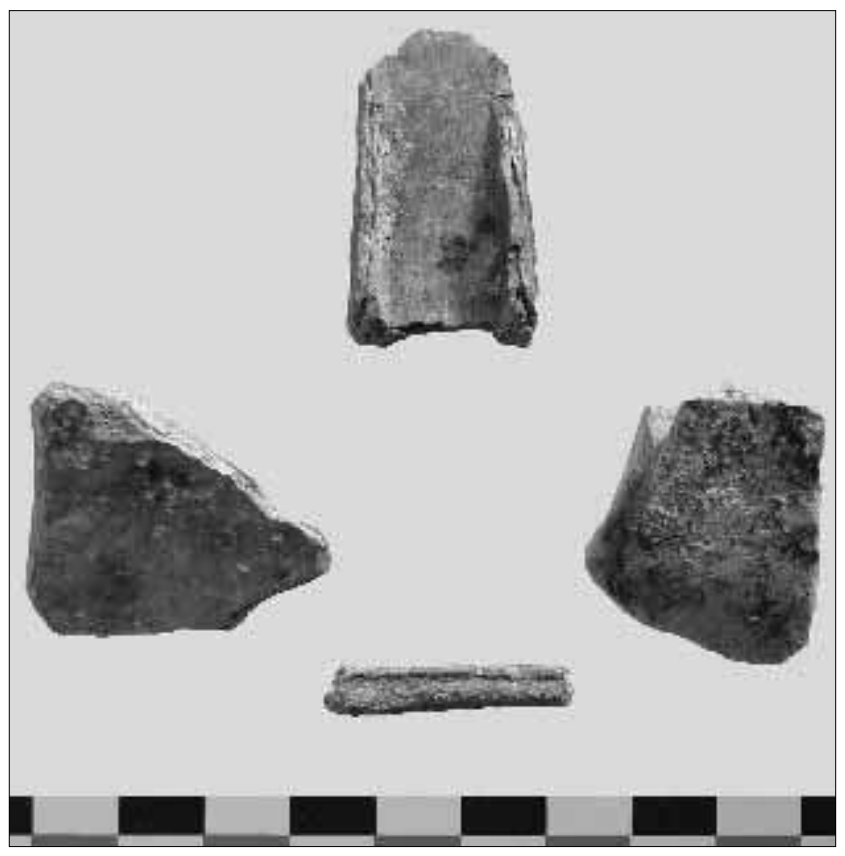

Figure 15 : Talon de hache $\mathrm{n}^{\circ} 4$ (en haut), fragments de tranchants $\mathrm{n}^{\circ} 5$ (à gauche) et $\mathrm{n}^{\circ} 7$ (à droite), tige rainurée $\mathrm{n}^{\circ} 8$ (en bas).

Figure 15: Palstave butt end $n^{\circ} 4$ (Top), palstave and flanged-axe blade fragments $n^{\circ} 5$ (Left) and grooved rod $n^{\circ} 8$ (Bottom).

- un fragment de tranchant de hache, probablement à rebord (fig. $13 \mathrm{n}^{\circ} 7$ ), dont le tranchant pourrait avoir été mis en forme par martelage;

- un segment de tige ou de manche marqué de deux rainures opposées longitudinales (fig. $13 \mathrm{n}^{\circ} 8$, fig. 15); il pourrait s'agir d'un élément de soie d'outil de type ciseau, rasoir ou spatule...;

- un fragment parallélépipédique indéterminé (non figuré).

\section{Conclusions}

\section{Caractérisation du dépôt}

Deux aspects caractérisent le dépôt de Keravel : la présence d'objets fragmentés ou usés d'une part et l'association d'éléments d'armement (pointe de lance) et d'outillage ou d'objets divers (haches, tige) d'autre part. Le nombre minimum d'objets conservés et identifiés est relativement faible (neuf) pour une masse totale de 505,5 g. Il a été montré que le dépôt a été effleuré par la fosse Us.01 et que dans ce secteur à forte pente, l'érosion naturelle et anthropique fut importante. Dès lors, la question se pose de savoir si les objets présentés ici constituent l'ensemble ou simplement une part du dépôt d'origine. Il apparaît cependant, à la lumière des synthèses récentes, que masse et effectif sont dans la moyenne des dépôts mis au jour dans le nord-ouest de la France (Gabillot, 2003).

Ses caractéristiques de composition apparentent assez bien le dépôt de Keravel à un ensemble d'objets destinés à la refonte, situation fréquente dans les dépôts appartenant à l'horizon de Tréboul. Une telle diversité est courante à l'âge du Bronze, notamment à partir du Bronze moyen, " période où la fabrication d'objets métalliques va largement se diversifier et s'amplifier" (Giot et al., 1995). Plusieurs dépôts de ce type sont recensés dans le nord Finistère, l'un des plus proches étant celui de Plouguin. Les communes environnantes de Plouguerneau, Ploudalmézeau et SaintFrégant (cf. infra, fig. 17) ne sont pas en reste, preuve de la densité géographique des dépôts dans le Léon (Galliou, 1989; Gabillot, 2003).

\section{Datation}

Plusieurs éléments permettent de dater le dépôt de Keravel, à commencer par la typologie des objets composant le lot : deux d'entre eux, la pointe de lance $\left(\mathrm{n}^{\circ} 1\right)$ et la petite hache ( $\left.n^{\circ} 3\right)$ appartiennent au groupe de Tréboul (fig. 16). Ce groupe typologique est daté du début du Bronze moyen (Briard, 1965).

Une datation par radiocarbone, obtenue sur un fragment du sac en toile de lin qui avait contenu les bronzes de Tréboul, propose une large fourchette chronologique calibrée entre 1730 et 1475 avant J.-C. (Roussot-Larroque in Le Bihan et al., à paraitre). Les différents fragments de talons et de tranchants présents à Lannilis s'accorderaient avec cette date et cette typologie, notamment le tranchant de hache à rebords. Par comparaison, un talon de hache, également de type Tréboul, mis au jour sur l'habitat de Mez-Notariou à Ouessant, est daté par la stratigraphie et le mobilier céramique d'un horizon antérieur à 1450 avant J.-C., entre 1650 (?) et 1450 avant J.-C. (Le Bihan et al., à paraître).

Seuls les fragments de la hache à talon de type breton $\left(n^{\circ} 6\right.$ $\mathrm{a}$ et b) sont un peu plus récents. Ce type de hache est daté de la seconde phase du Bronze moyen et du tout début du Bronze final (Briard et Verron, 1976). Il est difficile d'être plus précis compte tenu de l'aspect fragmentaire de l'exemplaire de Lannilis qui limite une attribution typologique plus poussée. La hache de Pouldergat, comparable par son décor, est attribuée à l'horizon de Tréboul par J. Briard (fig. 16), soit entre 1550 et 1400 avant J.-C. (Bronze moyen I). Enfin, les caractéristiques technologiques (net rentrant de la butée) évoquées pour les haches $\mathrm{n}^{\circ} 3$ et 6 (cf. supra) semblent plaider pour une datation avancée au sein du Bronze moyen.

La coexistence d'objets anciens ou archaïsant (type de Tréboul) et de haches de type breton, considérés comme 
Figure 16 : Planches de comparaison (d'après Briard, 1965 à gauche et en bas; d'après Giot et al., 1995 à droite).

Figure 16: Metalwork comparisons.

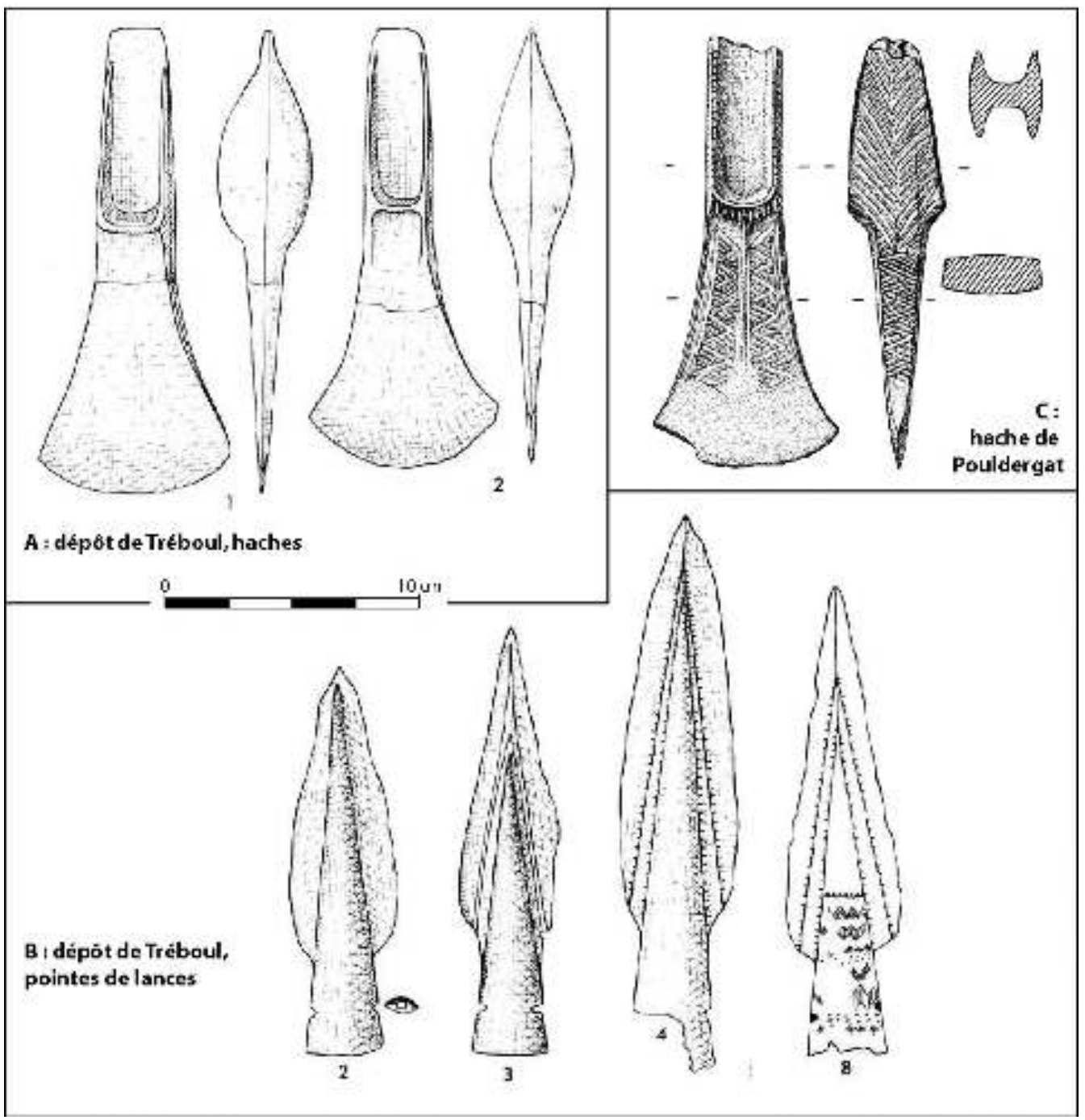

plus évoluées, est relativement fréquente, les dépôts constitués exclusivement de haches bretonnes étant plus récents (Briard, 1965; Gabillot, 2003). Citons pour exemple le dépôt de Saint-Gravé dans le Morbihan, proche par sa composition de celui de Lannilis (une pointe de lance de type Tréboul, une hache à rebord et une hache à talon de type breton), daté vers 1450/1400 avant J.-C.

En s'appuyant sur ces comparaisons, le dépôt de Lannilis Keravel daterait d'une période où la production des deux types de haches, Tréboul et breton, coexistaient. Par analogie avec les ensembles de Saint-Gravé et de Pouldergat, une datation autour du $\mathrm{xv}^{\mathrm{e}}$ siècle avant J.-C. peut être suggérée.

\section{Un dépôt typique de l'âge du Bronze armoricain}

Le phénomène des " dépôts " est général à toute l'Europe au Bronze moyen et se prolonge largement au Bronze final.
Si les objets en bronze ont fait leur apparition en Bretagne dès le Bronze ancien, ce n'est qu'au cours du Bronze moyen que se développent les fabrications d'objets métalliques en se diversifiant et s'amplifiant. Une première étape de ce dynamisme est matérialisée par les multiples dépôts du groupe de Tréboul. C'est surtout sous cette forme de dépôts enterrés que les objets (armes outillage, bracelets...) sont parvenus jusqu’à nous. Ces ensembles sont généralement interprétés comme artisanaux : réserves familiales, stocks de marchands ou récupération pour refonte selon la taille des dépôts, la qualité ou l'état des objets. Certains de ces dépôts, retrouvés au fond de cours d'eau, marais ou tourbières sont considérés comme votifs (Giot et al., 1995). Dans le cas de Keravel, la petite quantité de mobilier mis au jour ainsi que sa piètre qualité (fragments d'objets ou pièces très usées) plaident en faveur d'un lot destiné à la refonte. Cependant, sa localisation à proximité du ruisseau, en limite de zone marécageuse, pourrait lui conférer des caractères religieux. Si cette der- 
nière hypothèse doit être évoquée, elle ne semble pourtant pas devoir être retenue compte tenu de la modestie de ces éventuelles offrandes. Malgré tout, ce jugement qualitatif est un point de vue actuel : notre connaissance des religions de ces époques demeure encore lacunaire. Les découvertes des dépôts à caractères rituels de Mez-Notariou à Ouessant, avec leur association faune marine, sélection d'ossements de faune terrestre et objets métalliques (poignard, bracelets, épingle, rasoir et patelle en bronze), le montrent bien (Le Bihan et al., 2007, p. 15-37; Le Bihan et al., à paraître).

Les objets mis au jour dans le dépôt de Lannilis se rattachent pour la plupart à la typologie du groupe de Tréboul. Celle-ci regroupe des dépôts situés sur l'ensemble de la Bretagne, Loire-Atlantique incluse, dont plusieurs dans le nord Finistère comprenant des haches, des épées et poignards, de l'outillage (marteaux, ciseaux ou burins) mais peu d'objets de parure. Les productions de ce type, qui se développent au Bronze moyen, trouvent des similitudes aussi bien avec des ensembles normands ou britanniques que vers l'embouchure de la Loire ou de Gironde. Des objets relevant de cette typologie furent également découverts dans les bassins rhodanien et rhénan, preuve d'échanges entre tou- tes ces régions. Par ailleurs, les routes commerciales maritimes sont déjà fréquentées à ces époques. Pour la Bretagne, l'approvisionnement en cuivre destiné à la fabrication du bronze est essentiel; en contrepartie, l'étain du Léon (secteur de Saint-Renan) est un produit d'exportation. Pour cette partie nord du Finistère, l'île d'Ouessant, avec son habitat, son sanctuaire et son atelier de bronzier du Bronze moyen/ final I de Mez-Notariou, est le meilleur exemple à ce jour de l'interaction entre les populations locales et les échanges atlantiques mais également continentaux (Le Bihan $e t$ al., à paraître).

À Keravel, le dépôt demeure isolé, aucune autre structure de cette période n'ayant été découverte sur le site. Les éléments contemporains les plus proches sont les sépultures recensées sur le territoire de la commune. Ce dépôt complète une cartographie déjà dense des dépôts armoricains du Bronze moyen (fig. 17). Manquent encore les habitats pour connaître le mode de vie des artisans liés à ces dépôts de fondeurs. Les indices de peuplement (sépultures, dépôts, trouvailles d'objets isolés) sont pourtant nombreux pour ce secteur du Léon où l'environnement naturel est favorable à l'implantation humaine (terres cultivables, ressources mari-

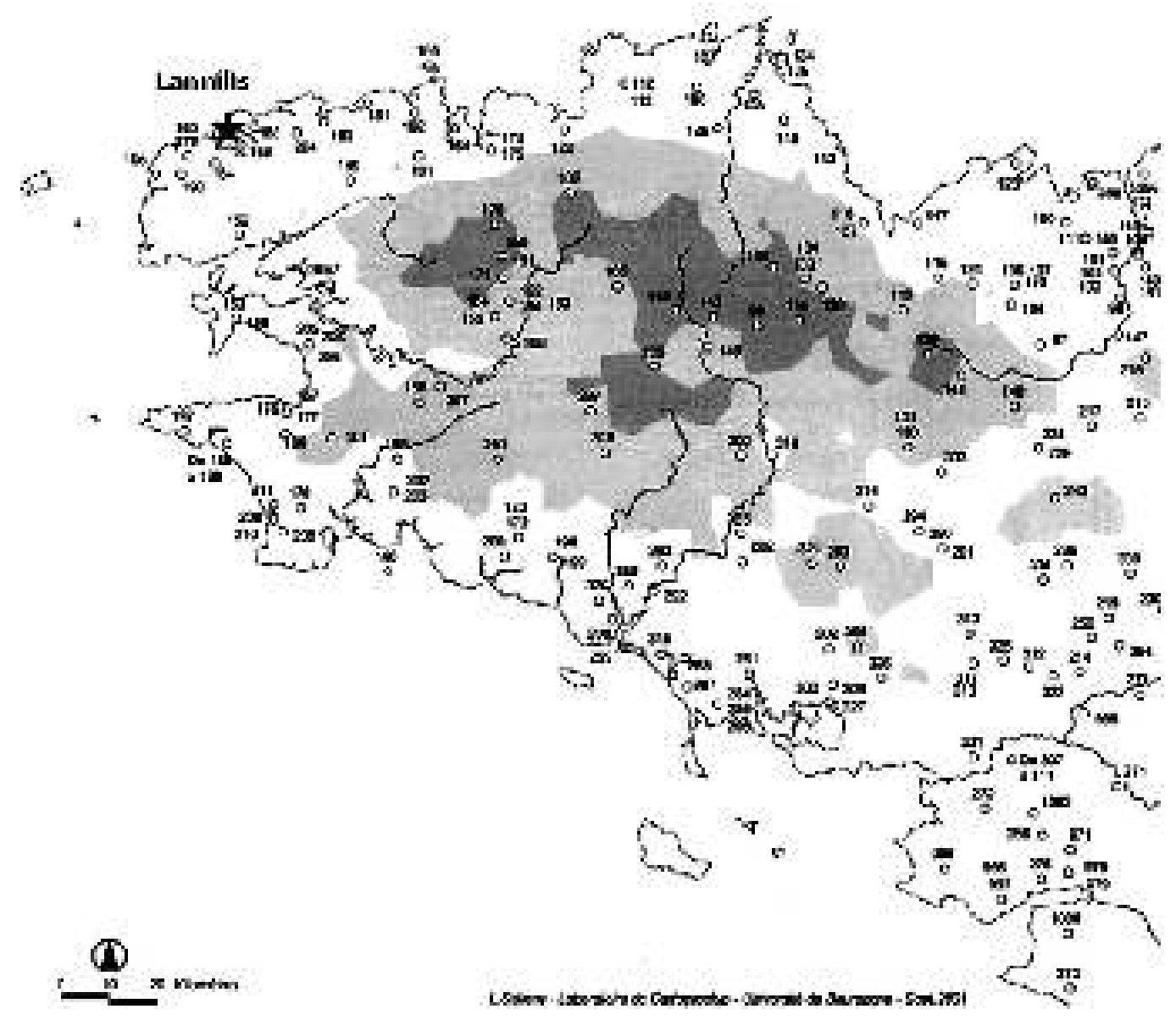

Figure 17: Carte des dépôts du Bronze moyen en Bretagne complétée par celui de Keravel (d'après Gabillot, 2003). Figure 17: Distribution map of Middle Bronze Age hoards in Brittany, including Keravel. 
times). Malgré quelques découvertes, liées aux opérations d'archéologie préventives, les lacunes sur l'habitat à l'âge du Bronze demeurent malheureusement générales dans la région.

\section{Bibliographie}

BRIARD, J., 1965 - Les dépôts bretons et l'âge du Bronze atlantique, Rennes, Travaux du laboratoire d'anthropologie de la Faculté des Sciences, $352 \mathrm{p}$.

Briard, J. et Mohen, J.-P., 1983 - Typologie des objets de l'âge du Bronze en France, fascicule II : poignards, hallebardes, pointes de lance, pointes de flèche, armement défensif. Paris, Société préhistorique française, $159 \mathrm{p}$.

Briard, J. et Verron, G., 1976 - Typologie des objets de l'âge du Bronze en France, fascicule III : haches (1), Paris, Société préhistorique française, $122 \mathrm{p}$.

Briard, J. et OnNée, Y., 1971 - Pointes de lances et haches décorées du Bronze moyen à Pouldergat et Tréboul (Finistère), Annales de Bretagne, 78, p. 25-36.

Gabillot, M., 2003 - Dépôts et production métallique du Bronze moyen en France nord-occidentale, Oxford, Hadrian books (BAR international Series, 1174), $471 \mathrm{p}$.

Gallou, P., 1989 - Carte archéologique de la Gaule, le Finistère (29). Paris, Académie des Inscriptions et Belles-Lettres, 229 p.

Giot, P.-R., Briard, J. et Pape, L., 1995 - Protohistoire de la Bretagne, Rennes, Ouest-France, 424 p.

Guilaine, J. (dir.), 1991 - Pour une archéologie agraire, Paris, Armand Colin, 576 p.

Le Bihan, J.-P., 1997 - Les vestiges des Hauts de Penvillers à Kerfeunteun (Quimper Finistère) (DFS), Rennes, SRA de Bretagne.

LE BiHAN, J.-P. (dir.), 2008 - Vestiges protohistoriques et antiques découverts à Mez-Notariou (Ouessant), (DFS de fouille programmée, 2006-2007-2008), Rennes, SRA de Bretagne.
LE Bihan, J.-P. et VILLARD, J.-F., 2001 - Un établissement rural gallo-romain au Moustoir 3 à Quimper (DFS de diagnostic archéologique), Rennes, SRA de Bretagne.

Le Bihan, J.-P. et VillaRD, J.-F., 2003a - Parcellaire gallo-romain et habitat du Moyen Âge au Moustoir 1 à Quimper (DFS), Rennes, SRA de Bretagne.

Le Bihan, J.-P. et Villard, J.-F., 2003b - Habitats de l'âge du Bronze au Moyen Âge au Moustoir 2 à Quimper (DFS), Rennes, SRA de Bretagne.

LE BiHAN, J.-P. (avec la collaboration de GUILLAUMET, J.-P., Meniel, P., Roussot-Larroque, J. et Villard, J.-F.), 2007 Du Bronze moyen à l'aube du Moyen Âge, un lieu de culte révélateur de la fonction insulaire atlantique : Ouessant MezNotariou. Aremorica, études sur l'ouest de la Gaule romaine, Brest, Université de Bretagne occidentale, CRBC,133 p.

Le Bihan, J.-P., et ViLlaRd, J.-F. à paraître - Archéologie d'une île à la pointe de l'Europe, tome 2: L'habitat de Mez-Notariou de origines à l'âge du Bronze, Rennes, coéd. CRAF/PUR.

Le Goffic, M., 2006 - Le caveau de l'âge du Bronze de Pratar-Simon Pella, Lannilis (Finistère) (DFS), Rennes, SRA de Bretagne.

Roy, E., 2008 - Diagnostic archéologique avant l'implantation d'une zone d'aménagement concerté au lieu-dit Kerjaouen à Quimper (Finistère) (Rapport de diagnostic archéologique), Rennes, SRA de Bretagne.

VILlaRD, J.-F., 2002 - Les vestiges archéologiques de Kerjaouen à Quimper (Finistère) (DFS de diagnostic), 2 volumes, Rennes, SRA de Bretagne.

-, 2005 - Les vestiges antiques et médiévaux du 22 de la rue Bourgles-Bourgs à Quimper : établissements périurbains et ruraux de l'époque gallo-romaine, carolingienne tardive et du bas Moyen Âge (DFS de fouille complémentaire), 2 volumes, Rennes, SRA de Bretagne.

—, 2007 - Le dépôt du Bronze moyen et les vestiges de parcellaire anciens et récents de Keravel en Lannilis, Finistère (DFS de diagnostic), Rennes, SRA de Bretagne. 
